\title{
Medienkompetent zum Schulübergang \\ Erste Ergebnisse einer Forschungs- und Interventionsstudie zum Medienumgang in der Frühen Bildung
}

Gudrun Marci-Boehncke, Matthias Rath und Anita Müller

\begin{abstract}
Das Mediennutzungsverhalten von Vierjährigen und ihrer Erzieherinnen in der Kita wurde in dieser Studie betrachtet. Über die Aussagen von Erzieherinnen und Eltern, aber auch qualitative Verfahren wurde erhoben, wie medienkompetent einerseits die Kinder eingeschätzt werden und welchen Medienumgang sie zeigen. Andererseits sollten auch die Erzieherinnen ihre Einstellungen zu Medien darstellen. Vor dem Hintergrund der Kapitalsorten- und Habitustheorie Bourdieus wurde ein Forschungs- und Interventionsprojekt konzipiert, das bei Erzieherinnen und Kindern gleichzeitig den integrierten Medienumgang fördert und über metakognitive Prozesse der Evaluation und Fortbildung die Einstellung zu Medien positiv beeinflussen soll. Ergebnisse der Studie aus dem ersten Projektjahr liegen vor, sie zeigen positive Veränderungen bei den Erzieherinnen in der Einschätzung der eigenen Fähigkeiten und denen der Kinder.
\end{abstract}

Bereits vor Schuleintritt geht in Deutschland eine Bildungsschere auf, die vor allem Kinder aus bildungsfernen Milieus und mit Migrationshintergrund betrifft (vgl. Becker u. Lauterbach 2004; Kreyenfeld 2004; Becker u. Biedinger 2006; Diefenbach 2007; Rabe-Kleberg 2010). Die Bildungsdefizite ziehen sich durch die gesamte Bildungsbiographie, bildungsnahe Milieus geben ihren Bildungsvorsprung an die nachwachsende Generation weiter, die Kinder und Jugendlichen bildungsferner Milieus, vor allem bei Migrationshintergrund, steigen mit einem objektiven Bildungsunterschied ein, den die Bildungsinstitutionen über die gesamte Bildungslaufbahn auch nicht kompensieren (vgl. Ehmke u. Jude 2010). So wird der Bildungsunterschied zur lebenslangen Bildungsbenachteiligung. Im Folgenden soll das Augenmerk auf die frühe Bildung gelegt werden, allerdings mit einer über diese Phase hinausweisenden Intention. Denn je früher Bildung gelingt, desto weniger werden herkunftsbedingte Bildungsunterschiede zum bildungsbiographischen Schicksal, wird der Schulübergang, wie Bildungsübergänge überhaupt, zur bildungsbiographischen Chance (vgl. Rath 2011) und nicht zum Spielball ethnischkultureller Disparitäten (vgl. Baumert u. a. 2010). Dies gilt auch für die Medienbildung. 
Verschiedene Studien zum Medienhandeln von Erzieherinnen und Erziehern (vgl. Six u. a. 1998; Six u. Gimmler 2007; Schneider u. a. 2010) zeigen die Bedingungen der Medienbildung in Kindertageseinrichtungen (vgl. Six 2010). Zum einen wird ein Zusammenhang mit institutionellen bzw. strukturellen Rahmenbedingungen der Einrichtungen und Kindergruppen deutlich - hierzu gehören Medien-, Finanz- und Personalausstattung und die Zusammensetzung der zu betreuenden Kindergruppen. Zum anderen ist Medienbildung abhängig von den individuellen Voraussetzungen der Erzieherinnen und Erzieher. Darunter fallen die medienpädagogische Ausbildung - sofern sie den Grundstein für das medienpädagogische Verhalten und Handeln der Erzieherinnen legt -, eine adäquate Vorstellung von Medienerziehung in der Kita sowie die eigene Medienkompetenz und Motivation, mit Medien in der Kita zu arbeiten. Erst wenn bei den Erzieherinnen und Erziehern die Einsicht vorhanden ist, dass Medienerziehung in der Kita notwendig, sinnvoll und umsetzbar ist, besteht die Chance, dass Medienerziehung in den Kita-Alltag integriert wird.

Das Forschungs- und Interventionsprojekt KidSmart zielt auf die Erfassung der Medienkompetenz der beteiligten Akteure im Prozess institutionalisierter frühkindlicher Mediensozialisation sowie auf die Begleitung eines strukturierten Interventionsprozesses, der direkt auf die Medienkompetenz der Kinder, aber auch die Medienkompetenz der Erzieherinnen ${ }^{1}$ zielt. In doppelter Weise unterscheidet sich KidSmart damit von den meisten bisherigen Untersuchungen zur Medienkompetenzförderung in der frühen Bildung:

1. KidSmart ist interventiv angelegt, das heisst, das Projekt greift in sein Forschungsobjekt ein, ermöglicht kontrollierte Massnahmen zur Veränderung und Verbesserung der Medienbildung in den untersuchten Einrichtungen. Dazu gehören materielle ebenso wie personelle und konzeptionelle Ressourcen.

2. KidSmart nimmt das gesamte Handlungsfeld der Frühen Bildung und seine Akteure in den Blick: die Erzieherinnen, aber auch die Kinder und die Eltern. Damit wird das Projekt der Tatsache gerecht, dass soziale Handlungssysteme multifaktoriell bestimmt sind, also jeder Handlungsfaktor das Gesamtsystem mit beeinflusst.

\section{Forschungsansatz}

Das KidSmart-Projekt ist konzipiert vor dem Hintergrund der Kapitalsorten- und Habitustheorie des französischen Soziologen Pierre Bourdieu (vgl. Bourdieu 1983; 1992). Bildung verstehen wir demnach als soziales Feld (vgl. Bourdieu 1987; Bourdieu u. Wacquant 1996), in dem jungen Menschen Bildungsangebote (hier Angebote zur Medienbildung) zum Erwerb inkorporierten kulturellen Kapitals gemacht

1 Im Folgenden wird bei der Beschreibung des Projekts sowie der Ergebnisse ausschliesslich die Form «Erzieherin» verwendet, da fast die gesamte Gruppe des befragten pädagogischen Personals aus Frauen bestand. Lediglich fünf Männer arbeiten in den Kitas. Sie sind jeweils mitgemeint. 
werden. Ein Ziel wäre z.B. die Institutionalisierung dieses Kapitals als Bildungsabschluss, das dann, transferiert in andere Felder (Schule, Berufsleben), soziales Kapital und symbolisches Kapital (z. B. Beruf) ermöglicht, die wiederum für den Erwerb ökonomischen Kapitals massgebend sind. Die Form dieser Transferierung sowie des Erwerbs von Kapitalien ist nach Bourdieu der Habitus (vgl. Bourdieu 1982). Unter Habitus versteht er die konkreten Dispositionen der Alltagskultur eines Handelnden, die er erworben hat und mit denen er in einem kulturellen Raum agiert. Das Repertoire solcher Habitus machen den Handlungsraum aus, über den ein Individuum verfügt. Da nicht das lernende Individuum allein massgebend ist, sondern das Feld, in dem es kulturelles Kapital inkorporiert, müssen auch die Akteure dieses Feldes - Institutionen, Personen - in den Blick genommen werden.

\section{Rahmendaten des Projekts}

Das Projekt mit einer Laufzeit von 2010 bis 2014 ist in Dortmund angesiedelt. Dafür spricht die besondere Situation des alten Industriestandortes. Dortmund hat einen Anteil von 29\% Einwohnern mit Migrationshintergrund sowie 12,8\% Arbeitslosen (vgl. Dortmunder Statistik 2011). Die beteiligten Kindertageseinrichtungen (Kitas) liegen grösstenteils im Dortmunder Norden, der in Bezug auf den Migrationsanteil (bis zu 63\%) ebenso wie in Bezug auf die Arbeitslosenquote (bis zu 24,9\%) weit über dem städtischen Durchschnittswert sowie über dem Landesdurchschnitt NRW liegt. In der ersten, hier vorgestellten Phase des Projekts (Abschluss September 2011) waren 21 Kitas beteiligt. Zwei weitere Phasen sind vorgesehen, in denen eine Weiterführung der ersten Phase in dann 33 Kitas sowie in die Schule hinein im Rahmen des offenen Ganztags im Grundschulbereich angedacht ist.

Organisatorisch ist das Projekt als Bildungsnetzwerk konzipiert (vgl. MarciBoehncke 2011), in dem eine Vielzahl von Institutionen zusammenarbeiten. Ein wichtiger Faktor, um die Kitas in Stand zu setzen, sich an einem Medienprojekt zu beteiligen, das über die klassischen Print-Medien hinausgeht, war die Versorgung der Kitas mit einer kindgerechten PC-Ausstattung. Hierfür konnte als externer Partner IBM Deutschland gewonnen werden, der im Rahmen seiner CSR (Corporate Social Responsibility)-Massnahmen inzwischen allein in Dortmund 100 KidSmart-Computer-Stationen zur Verfügung gestellt hat. Die KidSmart-Stationen bestehen aus einem normalen, netzfähigen PC mit Monitor, die beide in einem Kunststoff-Gehäuse robust und kindgerecht untergebracht sind (vgl. KidSmart 2001). Während des Projektverlaufs wurden die Kitas jeweils durch Lehramtsstudierende der Technischen Universität Dortmund unterstützt, die im Rahmen von Veranstaltungen und zusätzlichen Trainings auf diese Tätigkeit vorbereitet wurden. Weitere Partner sind der städtische Kita-Träger Fabido, das Dortmunder Systemhaus und Bits21. 
Das Projekt umfasst mehrere Teilziele und Zielgruppen, die organisatorisch und konzeptionell zusammengeführt werden. Zielgruppen sind die Erzieherinnen, deren Medienbildungskompetenz durch die Einbindung in die einzelnen Teilprojekte vor Ort und die Fortbildungsmassnahmen verbessert werden sollen, dann die Kita-Kinder, schwerpunktmässig aus bildungsfernem Milieu, deren Medienkompetenz im Hinblick auf aktiv-produktive und medienkritische Teilkompetenzen verbessert werden soll, die Eltern, deren Medienpraxis nicht nur erfasst, sondern denen auch zusätzliche Informationsangebote im Rahmen der Elternarbeit der jeweiligen Kita gemacht werden, und schliesslich die Lehramts-Studierenden, die im Rahmen der forschenden Lehre nicht nur Einblick in die Medienbildung der frühen Bildung bekommen, sondern auch eigene Medienbildungskompetenz erweitern und für ihre zukünftige Berufspraxis für die Medienkompetenzen und Defizite der Schulübergänger sensibilisiert werden.

In individuell erarbeiteten Projekten, die sich an den für die Kitas relevanten Bildungsstandards (vgl.MSJK NRW 2003; MSW NRW/MFKJKS NRW 2011) in Nordrhein-Westfalen orientieren, wird im Kontext lebensweltlich angebundener Themen ein breites Spektrum an kreativer Medienarbeit vermittelt. Die KidSmartStation stellt dementsprechend nur ein Medium unter vielen dar, die zur aktiven Gestaltung im Projekt mit den Kindern genutzt werden. Das Besondere des Projekts liegt zum einen in der Begleitforschung, die kontinuierlich in die Kitas zurückgespiegelt wird und damit die Möglichkeit zur metakognitiven Reflexion bietet. Zum anderen wird versucht, durch kontinuierliche Begleitung der Erzieherinnen in der selbstgestalteten Medienarbeit vor Ort durch medienkompetente Studierende Sicherheit und Nachhaltigkeit für die Erzieherinnen zu erreichen. Dahinter steht die Vermutung, dass Erzieherinnen über eine kontinuierliche und niederschwellige Unterstützung von Studierenden in den Einrichtungen nachhaltiger fortgebildet werden als über zentrale Fortbildungsangebote allein, die nicht in die tägliche Arbeit hineinreichen.

\section{Methodisches Vorgehen}

Das Forschungsprojekt ist in die qualitative Forschung einzuordnen. Wissenstheoretisch ist die Studie im Bereich der Heuristischen Sozialforschung (vgl. Glaser u. Strauss 1998; Kleining 1994; Krotz 2005) anzusiedeln. Auf der Daten- und Methodenebene wird ein komplexes, mehrperspektivisches Forschungsdesign (vgl. Flick 2004; 2005) angewendet. Um möglichst viele Perspektiven auf die Medienpraxis im privaten Kontext sowie im Kita-Alltag zu gewinnen, werden sowohl Eltern als auch Kinder und Erzieherinnen befragt. Als Methoden zur Datengewinnung werden halbstandardisierte Fragebögen, qualitative Interviews und teilnehmende Beobachtung eingesetzt. Die Erhebung der Daten erfolgt zu unterschiedlichen 
Messzeitpunkten: vor Projektbeginn, während der Interventionsphase und nach Abschluss der einzelnen Projektphasen.

Die Variation der verschiedenen Perspektiven gibt Auskünfte über Eltern, Erzieherinnen und Kinder. In der ersten Projektphase wurden 21 Kitas betrachtet. 175 Erzieherinnen wurden zu ihrer eigenen Mediennutzung und Medienkompetenz schriftlich befragt. 269 Elternteile wurden über das Medienverhalten von insgesamt 307 Kindern (im Alter zwischen 4 und 5 Jahren) und der Mediennutzung im familiären Kontext konsultiert, wobei der Fragebogen in sieben Sprachen zur Verfügung stand. Ebenfalls wurden die am Medienprojekt beteiligten Erzieherinnen zu denselben Kindern befragt. In jeder Kita werden von der Forschergruppe zwischen vier und acht leitfadengestützte Interviews geführt. Somit wird über ein Drittel der am Projekt beteiligten Kinder mündlich zu seinen Medienvorlieben befragt werden. Um eine künstliche Interview-Situation zu umgehen, wird durch den Einsatz einer Handpuppe das Interview in eine natürliche Spielsituation der Kinder gewandelt (vgl. Weise 2008; 2011). Die Fragebögen und Puppet Interviews werden durch die Methode der «teilnehmenden Beobachtung» (vgl. Mikos 2005) ergänzt. Ihr Schwerpunkt liegt auf dem Umgang der Kinder mit «klassischen» und «neuen» Medien, dem Sozialverhalten und der Entwicklung von Kompetenzen.

\section{Darstellung der vorläufigen Forschungsergebnisse}

\section{Institutionelle Rahmenbedingungen}

Auf die Frage, worin die Erzieherinnen die grössten Hindernisse für ihre pädagogische Tätigkeit allgemein sehen, nannten sie am häufigsten strukturelle Rahmenbedingungen wie die Erzieher-Kind-Relation bzw. den Personalschlüssel nach dem Kinderbildungsgesetz NRW (KiBiz), Zeitmangel sowie das Fehlen ökonomischer Ressourcen (Ausstattung, finanzielle Mittel). Darüber hinaus wurden die Verständigungsprobleme mit Eltern und Kindern angesprochen, die vorrangig auf mangelnden Deutschkenntnissen der Eltern und Kinder beruhen. Von einigen Erzieherinnen wurde auch die Kooperation mit Kollegen und Kolleginnen sowie gemeinsame Absprachen als schwierig bewertet. Speziell in Bezug auf Medienbildungsmassnahmen erschweren zudem bei einem Teil der Befragten der eigene Kompetenzmangel sowie Desinteresse auf Seiten der Eltern die Arbeit. In erster Linie werden deshalb Veränderungen in Bezug auf strukturelle Bedingungen gefordert, die sich v. a. auf kleinere Gruppengrössen und Personalzuwachs beziehen. Ebenso wünschen sich die Erzieherinnen mehr Vor- und Nachbereitungszeit sowie eine bessere Ausstattung in Bezug auf die Medienarbeit (8,8\%).

Analog zu den Hindernissen und Wünschen für die pädagogische Arbeit lassen sich die Antworten zu den Idealbedingungen betrachten, die nach Angaben der Erzieherinnen für die Arbeit mit Medien erforderlich sind. Zeit, genügend Personal 
und eine gute Ausstattung werden von den Erzieherinnen an dieser Stelle vorausgesetzt, um medienpädagogische Arbeit leisten zu können. Als noch relevanter wird jedoch eine Vertiefung der eigenen Medienkompetenz, v. a. in den Bereichen Technik und Fachwissen, durch Fortbildungen und Begleitung angesehen.

Betrachtet man die Angaben zu den vorhandenen Medien in den Kitas, zeigt sich, dass die Einrichtungen insgesamt medial schon relativ breit aufgestellt sind. Jeder Kita stehen mindestens ein Computer und ein digitaler Fotoapparat für die Medienarbeit mit Kindern zur Verfügung. Die Hälfte ist mit einem TV-Gerät ausgestattet und alle Einrichtungen verfügen über Bücher und Hörspiele. Der grösste Mangel besteht an Beamern und OH-Projektoren. Jedoch zeichnen sich in den Angaben der Erzieherinnen innerhalb derselben Kita grosse Diskrepanzen ab. So stehen entweder nicht alle Medien jeder Erzieherin zur Medienarbeit mit den Kindern zur Verfügung oder nicht alle Erzieherinnen sind über die Medienausstattung in ihrer Kita informiert.

Soziales Feld «Kindertageseinrichtung»

- Vorerfahrungen und Vorwissen im medienpädagogischen Handlungsfeld Die Mehrheit der Erzieherinnen (74\%) hat vor KidSmart noch keine Medienprojekte durchgeführt. Es wurde zwar eine relativ grosse Anzahl an Projekten angegeben, jedoch konnte ein Teil der Antworten nicht als Medienprojekt gewertet werden (vgl. Projektkategorien in Katz u. Chard 2000). Fast $20 \%$ aller Erzieherinnen haben sich vor dem Projekt noch nie mit Medienerziehung auseinandergesetzt, darunter $13 \%$ der am Projekt aktiv beteiligten Erzieherinnen. Über die Hälfte der befragten Erzieherinnen (53\%) haben im Rahmen ihrer Ausbildung Medienerziehung nicht kennengelernt.

- Bildungsauftrag Medienerziehung

Zur Frage nach den wichtigsten Themen in der Kita nennen nur 9,7\% der befragten Erzieherinnen Medienerziehung. Akzentuiert werden Sprachförderung (79,4\%), soziales Lernen (70,3\% ) und Bewegungserziehung (53,7\%). Gerade in NRW basiert dieses Bewusstsein für Sprachförderung sicher auch auf dem verpflichtend eingeführten Sprachdiagnoseverfahren (Delfin 4) und den entsprechenden Förderprogrammen. Eine Öffentlichkeit für diesen Förderbereich ist in der Frühen Bildung erfolgreich hergestellt worden. Für Medienbildung ist dies offensichtlich nicht der Fall (vgl. Abb. 1) 


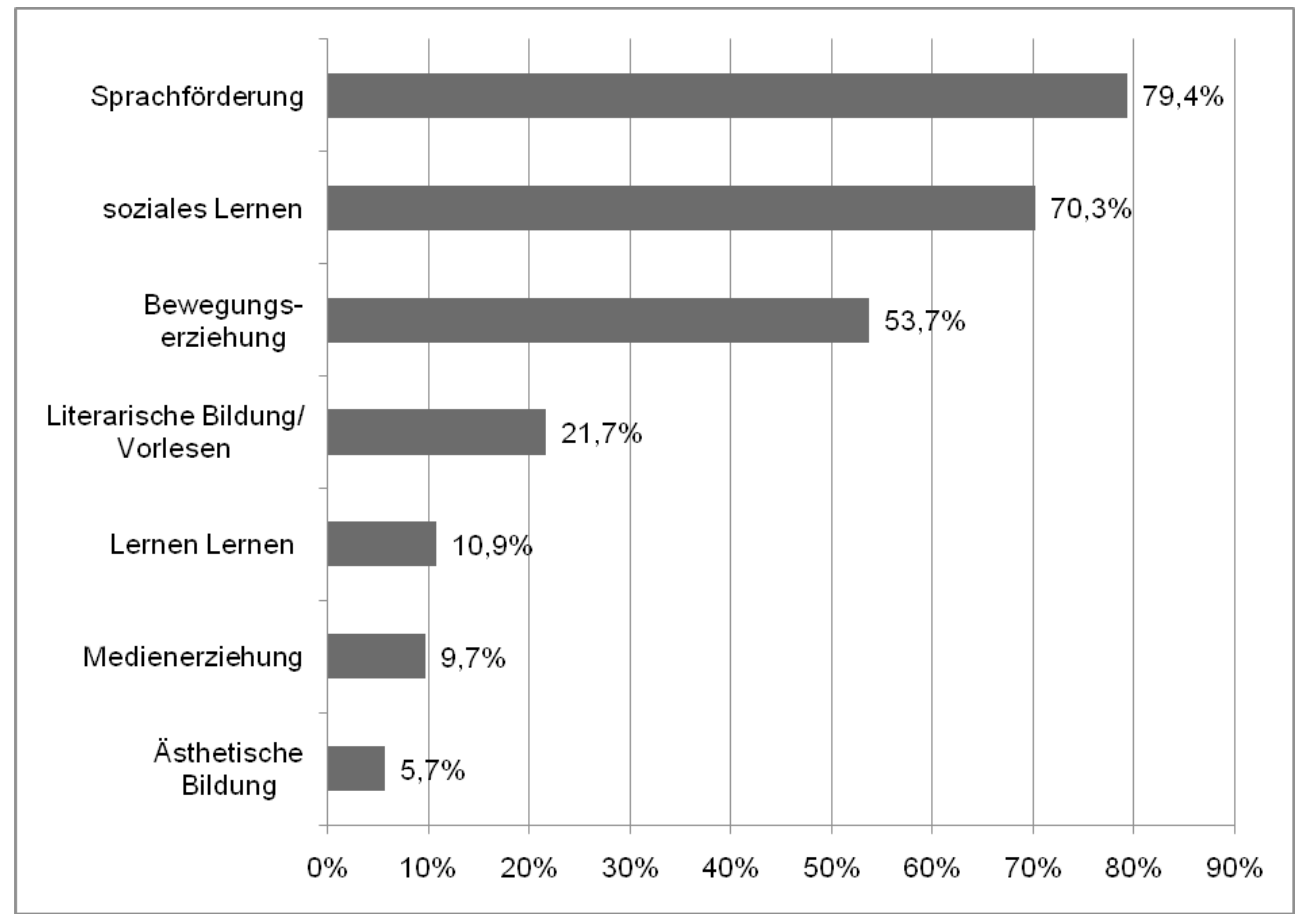

Abbildung 1: Wichtigstes Thema in der Kita-Arbeit für die Erzieherinnen ( $N=175)$

Vergleichbare Ansichten spiegeln sich auch bei den Eltern wider. Nur 10,3\% der Eltern halten Medienerziehung für eines der wichtigsten Themen in der Kita. Auch die Eltern legen den grössten Wert auf Sprachförderung und -diagnostik (70,8\% ). Bei der offen gestellten Frage, inwiefern Medienerziehung curricular in der Bildungsvereinbarung (MSJK NRW 2003) des Landes NRW verankert sei, fallen die Antworten der Erzieherinnen sehr unterschiedlich und unpräzise aus. Es wird häufig nicht ersichtlich, ob sie mit der Bildungsvereinbarung in Bezug auf Medienerziehung vertraut sind.

- Selbsteinschätzung der Qualifiziertheit im medienpädagogischen Kontext Ziel der Intervention des Projekts ist neben der Medienkompetenzvermittlung an die Kinder auch die Verbesserung der Medienbildungskompetenz der Erzieherinnen. ${ }^{2}$ Dabei sind zwei Bereiche zu unterscheiden: Zum einen geht es um die technischen Kompetenzen im Umgang mit Medien, zum anderen um die konzeptionelle Frage, wie Medien im Sinne einer «integrierten Medienerziehung» in die

2 Im Folgenden wird zwischen Antworten aller Erzieherinnen in den beteiligten Kitas und den aktiv im Projekt tätigen Erzieherinnen unterschieden. Die beteiligten Kitas haben jeweils eine Projektgruppe zusammengestellt, die von meist zwei Erzieherinnen betreut wurde, die dann auch an den Fortbildungen teilgenommen und die Interventionen mit den Kindern vor Ort durchgeführt hat. 
tägliche Kita-Arbeit, die nicht auf Medien als Thema ausgerichtet ist, einbezogen werden. Dieser letzte Aspekt ist besonders wichtig, da hier Medien als Form des Bildungsangebots allgemein berücksichtigt werden, unabhängig von der konkreten Bildungsthematik.

Vor der Intervention fühlten sich lediglich $45 \%$ der Erzieherinnen konzeptionell sicher hinsichtlich der Medienintegration in die Kita-Arbeit. Bei den Erzieherinnen, die aktiv am Projekt mitarbeiten und auch an den projektinternen Fortbildungen teilnahmen, waren es hingegen 62,5\%. Dies zeigt, dass diejenigen Medienbildung aktiv zu ihrer Sache machen und dafür notwendige Kompetenzen erwerben, die schon eine gewisse Vertrautheit mit dem Bereich aufweisen. Damit wird die besondere Bedeutung deutlich, Medienbildung bereits in die Ausbildung der Erzieherinnen zu integrieren.

Am Ende der ersten Projektphase hat sich dieser Anteil der Erzieherinnen und Erzieher, die sich sicher fühlen, Medien in die tägliche Kita-Arbeit zu integrieren, bei den aktiven Projekt- und Fortbildungsteilnehmerinnen von 62,5\% auf 75\% erhöht. Im Bereich der technischen Medienkompetenz gaben in der Erstbefragung 45\% aller Befragten an, sich als unzureichend kompetent zu erfahren. Nach der ersten Projektphase schätzen sich 70\% der aktiv am Projekt und den Fortbildungen teilnehmenden Erzieherinnen als technisch kompetent ein.

- Einstellung gegenüber Medien in der Frühen Bildung

Bei einem gewissen Teil der Erzieherinnen liess sich vor dem Projekt eine bewahrpädagogische Einstellung feststellen. 17,4\% der Erzieherinnen vertraten die Überzeugung, dass die Kita einen «medienfreien Raum» darstellen sollte. 16,7\% der Erzieherinnen, die aktiv am Projekt mitarbeiten, und $16 \%$ der Erzieherinnen, die nicht aktiv involviert sind, vertraten diese Auffassung (keine statistisch signifikante Abweichung). Bei keiner Erzieherin, die aktiv am Projekt mitwirkte, ist diese Ansicht nach der Intervention noch vorhanden.

Vor der Intervention herrschte bei 49,1\% aller Erzieherinnen die Befürchtung, dass sich Medien langfristig negativ auf die Gesundheit der Kinder auswirken könnten. Hier ergibt sich allerdings ein interessanter Unterschied, wenn wir diese Daten nach der späteren Projektbeteiligung differenzieren (vgl. Abb. 2). 


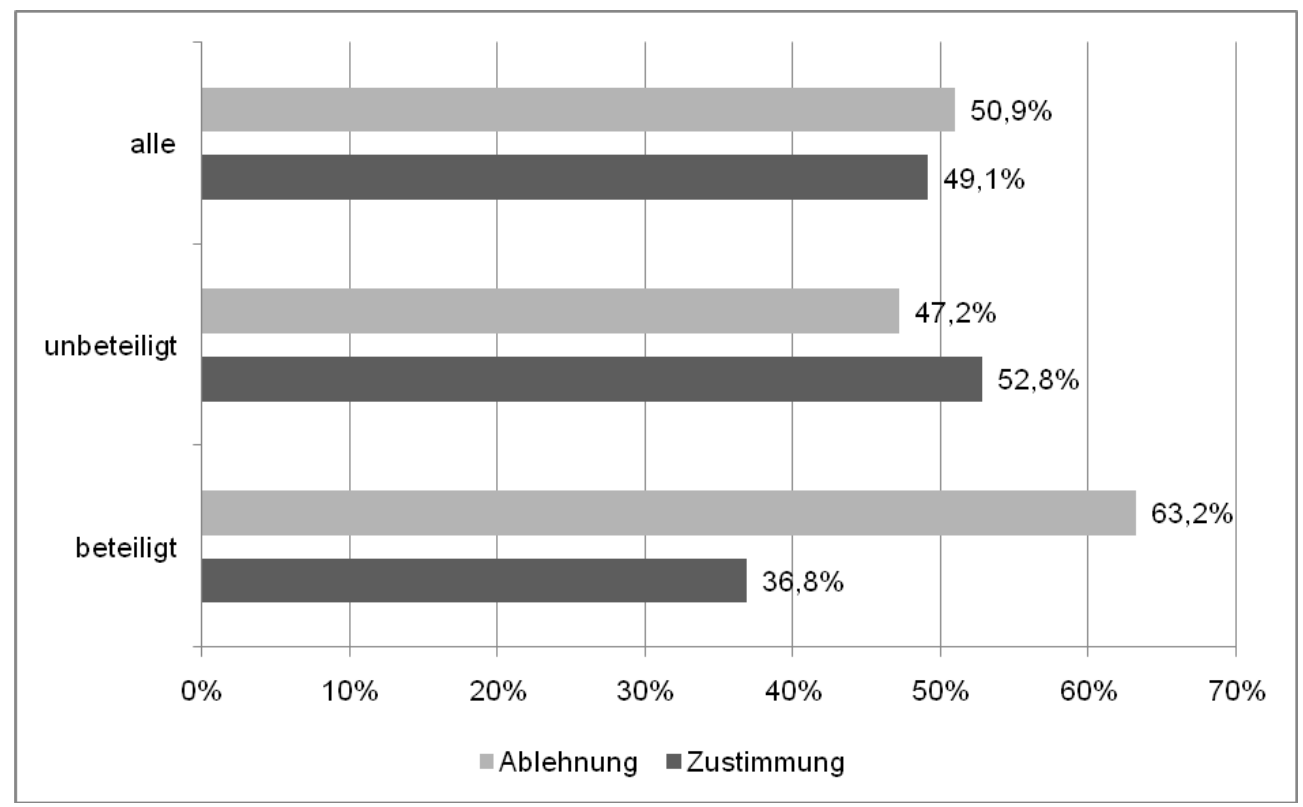

Abbildung 2: Stellungnahme der Erzieherinnen zu der Aussage: «Neue Medien wirken sich langfristig negativ auf die Gesundheit der Kinder aus», vor Durchführung des Projekts $\left(\mathrm{N}_{\text {alle }}=169 ; \mathrm{N}_{\text {beteiligt }}=38 ; \mathrm{N}_{\text {unbeteiligt }}=125\right)$

Hier zeigt sich bereits eine grundsätzliche Problematik bei kompetenzerweiternden Interventionen in einem auch weltanschaulich aufgeladenen Themenfeld wie «Medienarbeit in der Kita»: Die aktiv ansprechbaren und engagierten Pädagoginnen und Pädagogen (in allen Bildungsinstitutionen) sind diejenigen, die bestimmte Werthaltungen, die häufig Medienbildungsmassnahmen in einer Institution erschweren oder verunmöglichen, in einem geringeren Ausmass vertreten. Das heisst aber auch, dass diejenigen, die im Rahmen intervenierender Projekte angesprochen werden sollten, sich dem Projekt entziehen und somit Erfahrungen, die eine bewahrpädagogischen Haltung relativieren könnten, nicht machen. In gewissem Masse muss man bei Medienbildungsprojekten realistischerweise häufig von einer «Heilung der Gesunden» sprechen.

Bei den Erzieherinnen, die nach der ersten Projektphase zu der Aussage «Neue Medien wirken sich langfristig negativ auf die Gesundheit der Kinder aus» befragt wurden, tendiert nur noch eine der am Projekt beteiligten Erzieherinnen zu dieser Annahme (5,3\%) (vgl. Abb. 3). 


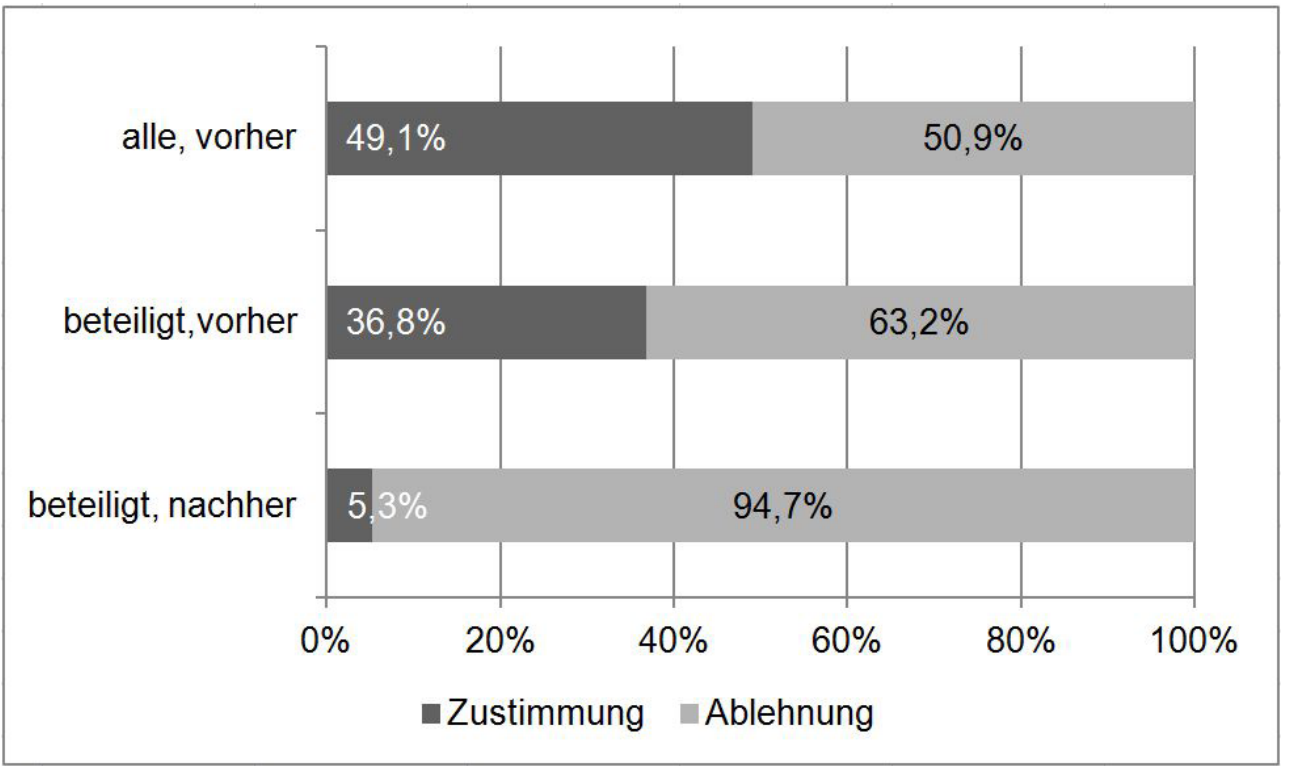

Abbildung 3: Stellungnahme der Erzieherinnen zu der Aussage: «Neue Medien wirken sich langfristig negativ auf die Gesundheit der Kinder aus» (Nalle, vorher=169; Nbeteiligt, vorher=38; Nbeteiligt, nachher=19)

- Motivation und Kompetenz, mit neuen Medien zu arbeiten

83,6\% aller Erzieherinnen der beteiligten Kitas haben von Beginn an Interesse mit neuen Medien in der Kita zu arbeiten. Von den Erzieherinnen, die sich im Projekt aktiv an Fortbildung und Intervention beteiligten, waren es vor Projektbeginn sogar $94,7 \%$. Vor dem Projekt zeigt sich, dass weniger als die Hälfte der Erzieherinnen, die am Projekt aktiv teilnehmen, in der Lage ist, aufwendigere Arbeiten, die mehrere Medien miteinander verbinden, durchzuführen. Vergleicht man die aktiv im Projekt tätigen Erzieherinnen mit den Erzieherinnen, die nicht an der Fortbildung und Intervention direkt teilnehmen, so fällt auf, dass die Multiplikatoren schon vor Projektbeginn kompetenter sind. Vorlesen und Inszenieren gelingt über $95 \%$ aller Erzieherinnen. Aber sowohl Mediengestaltungsprozesse mit tertiären Medien als auch solche in der Umgebung von Web 2.0 stellen eine besondere Herausforderung dar und können ohne zusätzliche Fortbildungen von über der Hälfte der Erzieherinnen nicht selbstständig geleistet werden. Nach der ersten Projektphase können fast alle der beteiligten Erzieherinnen Textverarbeitung leisten sowie Fotos digital aufnehmen (je $95 \%$ ) und bearbeiten (90\%).

- Mediennutzung während und ausserhalb der Projektarbeit

In den KidSmart-Projekten der einzelnen Kindertageseinrichtungen wurde ein breites Medienspektrum genutzt. Zu den am häufigsten eingesetzten Medien zäh- 
len Computer offline, Internet, Drucker, Bücher und Digitalkamera. Am liebsten wurde der Computer von den Kindern in der Peer Group genutzt. Vor allem Kinder mit einem anderen kulturellen Hintergrund als Deutsch sassen häufiger mit anderen Kindern an der Station (63\%). Bei Kindern deutscher Herkunft liegt die kooperative Nutzung um 10\% -Punkte niedriger. Auch liessen sich Kinder mit einem anderen kulturellen Hintergrund als Deutsch eher helfen als ihre Spielkameraden ohne Zuwanderungsgeschichte. Die durchschnittliche Zeit, die sich die Kinder mit der Station beschäftigten, lag bei weniger als einer halben Stunde pro Beobachtungstermin.

\section{Soziales Feld «Familie»}

- Mediennutzung in der Familie

Viele Medienhandlungen der Kinder finden im familiären Kontext statt. Daher haben wir die Eltern ( $\mathrm{N}=269)$ nach den Mediengewohnheiten und familialen Medienpraxen gefragt. Allerdings ist es sinnvoll, bevor wir uns die Daten zur Mediennutzung der Kinder genauer ansehen, die Freizeitbeschäftigung der Kinder allgemein in den Blick zu nehmen: Die Eltern machen bei den liebsten Freizeitbeschäftigungen der Kinder gendertypische Angaben, die auch statistisch hoch signifikant sind. Über alle Unterschiede hinweg werden die Themenorientierung und die Bewegungsorientierung der Kinder deutlich. Medien, hier vor allem die rezeptiven Medienangebote Fernsehen, Video und DVD, kommen erst auf dem 5. (Jungen) bzw. 4. (Mädchen) Rang.

Anders sieht es hingegen aus mit den Beschäftigungen, denen das Kind alleine, also ohne Freunde oder Geschwister, nachgeht. Hier werden rezeptive Medien (Fernsehen/DVD/Video) mit 35,6\% verhältnismässig hoch auf Rang 2 bewertet. Dies bestätigt die Ergebnisse unserer bisherigen Forschung (Marci-Boehncke u. Rath 2007b), dass Medien auch als soziale Substitute dienen. Die Gleichaltrigengruppe wird jedoch dem Medium vorgezogen, es sei denn, das Medium wird in der Gruppe selbst genutzt.

Blicken wir nun auf die spezifischen Fragen nach den Medienpraxen der Kinder. Zunächst die gemeinsame Mediennutzung in der Familie (vgl. Abb. 4): Erwartbar ist die Nutzung des Fernsehgeräts mit 82,6\%. Verhältnismässig hoch und mit unserer bisherigen Kita-Forschung kompatibel ist die Nutzung von Büchern mit 70,6\% . Nur jedes fünfte Kind spielt mit einem Familienmitglied Computer- und Konsolenspiele. 


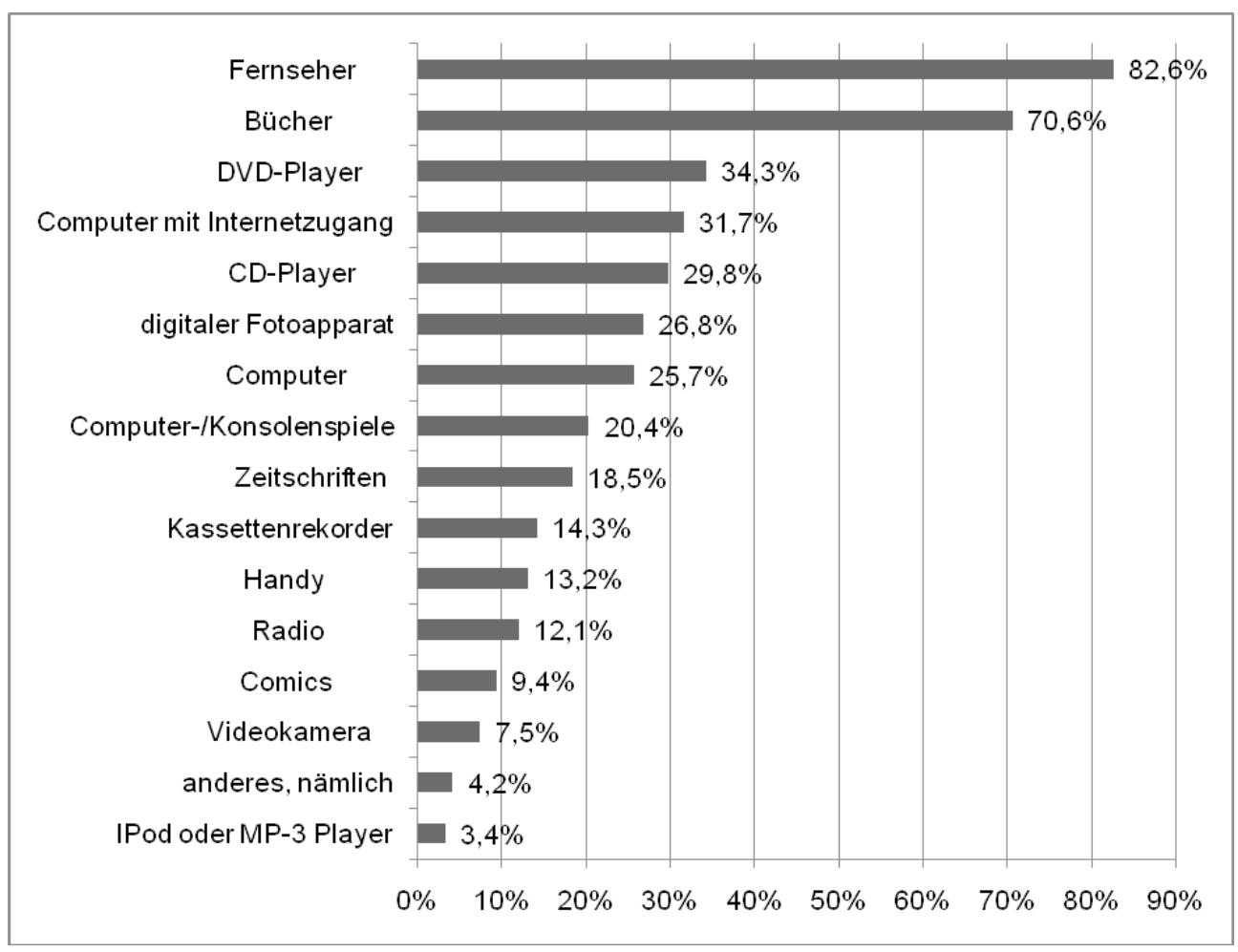

Abbildung 4: Eltern: gemeinsame Mediennutzung in der Familie (N=269)

Nehmen wir nun die Mediennutzung in den Blick, die Kinder zeigen: Bei den Medien, die die Kinder zu Hause nutzen, steht der Fernseher (90,7\%) auf dem ersten Platz, knapp gefolgt von Büchern (64,5\%). Ins Internet gehen bereits $29,1 \%$ der untersuchten 4- bis 5-jährigen Kinder. Fasst man die Antworten zum Computer zusammen (offline, online), so liegt der PC bereits bei 39,3\% .

Bei dem Lieblingsmedium der Kinder steht nach Auskunft der Eltern an erster Stelle der Fernseher (54,2\%), gefolgt vom Buch $(24,2 \%)$ und dem Computer mit Internetzugang (11,7\%). An fünfter Stelle liegen mit 10,2\% Computer-/Konsolenspiele. Also auch hier haben Bücher mit dem zweiten Rang noch einen verhältnismässig hohen Stellenwert. Soziale Erwartung muss hier als mögliche Antwortmotivation der Eltern einkalkuliert werden.

In den Familien ist die Medienkompetenzverteilung häufig zugunsten der Kinder verschoben. So geben $21,6 \%$ der Elternteile an, dass ihr Kind den Computer besser nutzen kann als sie selbst. 6,9\% der Kinder finden sich im Internet besser zurecht als die Erziehungsverantwortlichen. Bei Computer- und Konsolenspielen sind es sogar 35,3\% . 13,8\% der Kinder können, nach Angaben der Eltern, das Handy besser bedienen als sie selbst. 
- Medienerziehung in der Familie

Auf die Frage, inwiefern Medien ein Thema in der Familie sind, haben 4,8\% der befragten Elternteile nicht geantwortet. Von den antwortenden Eltern gaben $52,7 \%$ an, ihr Kind bei der Mediennutzung zu begleiten. 24,2\% der Eltern setzen Medienverbote als Erziehungsmittel ein, für 13,7\% der Eltern sind Medien überhaupt kein Thema und 7,4\% der Eltern überlassen die Medienorientierung ihres Kindes den älteren Geschwistern, von denen es die Mediennutzung und Medieninhalte übernimmt.

Allerdings sind diese Ergebnisse mit Vorsicht zu lesen. "Verantwortungsvolle Medienerziehung» ist als reale oder auch nur erwartete Erwartung Teil des öffentlichen Diskurses, vor allem nach vermeintlich durch Medien induzierten Gewalttaten von Kindern und Jugendlichen. Es ist daher nicht auszuschliessen, dass auch ein Teil dieser Antworten nicht die reale familiale Situation abbildet, sondern diese soziale Erwartung bedient.

- Medienhelden

70,8\% der Jungen und 66,4\% der Mädchen haben einen festen Medienhelden. Der Anteil der Kinder, die sich explizit Merchandising-Produkte mit Medienbezug wünschen, ist sogar höher, nämlich 76,9\%. Dies könnte darauf deuten, dass Konsumwünsche nicht nur direkt über die Mediennutzung, sondern auch mittelbar über die Peer Group-Kommunikation induziert werden. Spiderman, Barbie, Spongebob, Caillou und Hannah Montana sind momentan die beliebtesten KitaHelden. Bereits $42 \%$ der Kinder verfolgen ihre Helden konvergent, d. h. sie nutzen mehr als ein Medium, in dem ihr Held/ihre Heldin vorkommt. 60,2\% der Kinder bringen ihre Medienhelden in Form von Medienaccessoires in die Kita mit.

- Medienreaktionen

In der öffentlichen Diskussion um Mediennutzung von Kindern und den Wirkungen der Medien auf kindliches Verhalten werden häufig negative Auffälligkeiten benannt. In Übereinstimmungen mit früheren Studien (Marci-Boehncke u. Rath 2007b; Marci-Boehncke u. a. 2009) zeigen sich in lebensweltlichen Situationen nach Auskunft der Eltern bemerkenswerte Reaktionen (vgl. Abb. 5). So beobachtet nur ein geringer Teil der Eltern eine ängstliche oder aggressive Reaktion ihres Kindes auf Medien. Hingegen stellen Konsumwünsche in Bezug auf mediale Angebote die häufigste Medienreaktion dar, dicht gefolgt von Anschlusshandlungen des Kindes auf Medienrezeption, z. B. Nachspielen der Szenen, Malen oder Nachbauen, Weiterfantasieren. 


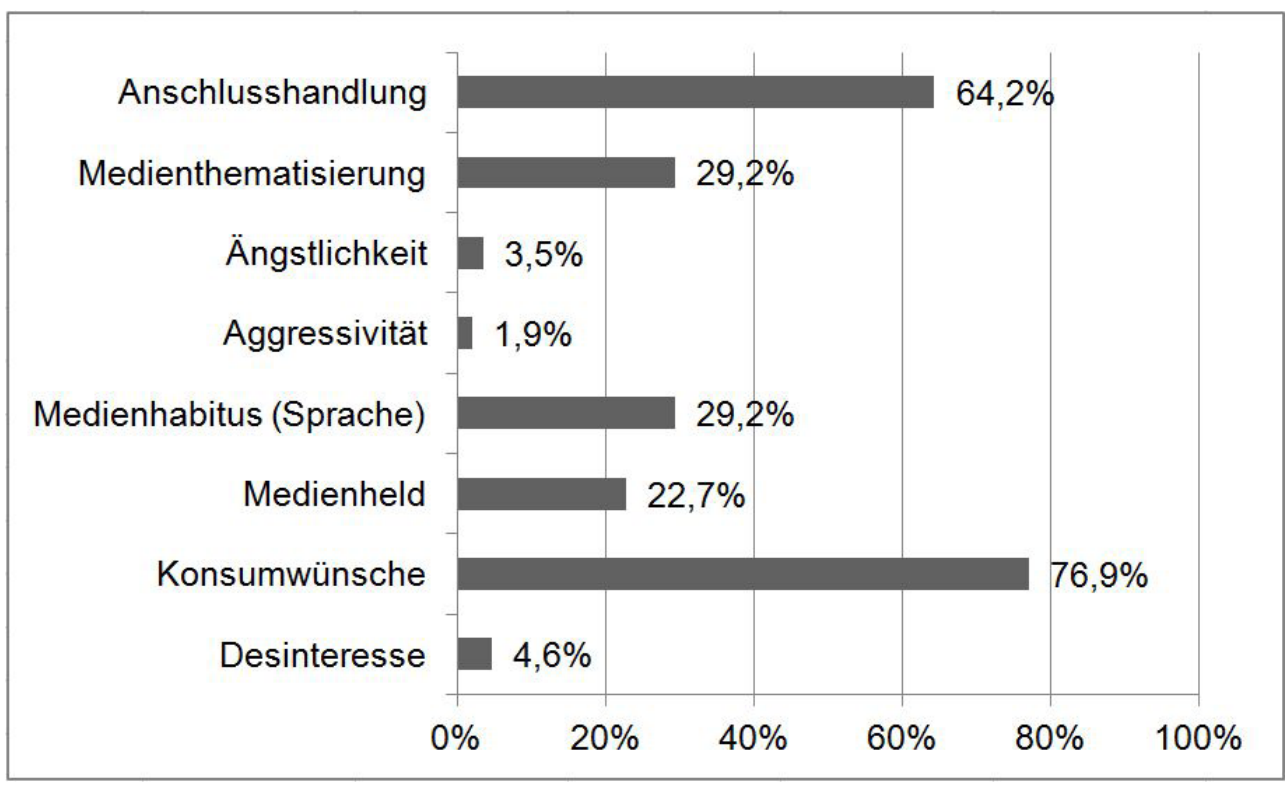

Abbildung 5: Beobachtete Medienreaktion der Kinder nach Auskunft der Eltern ( $N=260)$

An diesen beiden Punkten sollte medienerzieherisch besonders angesetzt werden, an den Anschlusshandlungen als geeigneter Form, Mediennutzung in der Kita zu thematisieren und an den Konsumwünschen als besonderer Herausforderung, den Kindern die ökonomischen Zusammenhänge der Medienangebote (z. B. Werbung) nahezubringen.

- Motivation und (Medien-)Kompetenzen Kinder

Die Motivation der Kinder im Projekt war sehr hoch. Vor der Projektarbeit wurde von den Erzieherinnen das Interesse bei 82,6\% der Kinder als hoch eingeschätzt, danach bei $85 \%$. Nach Aussagen der Eltern haben 98,1\% aller Kinder gerne am Projekt mitgewirkt und über $43,4 \%$ von ihnen haben darüber zu Hause berichtet. Bei über der Hälfte $(59,5 \%)$ konnte seither ein verstärktes Interesse am Medium Computer, bei 32,1\% an der Digitalkamera und bei 15,5\% an Büchern beobachtet werden.

Ein Grossteil der Kinder (90\% laut Erzieherinnen, 84\% laut Eltern) hat durch die aktive Projektarbeit an Kompetenzen hinzugewonnen. Darunter fallen sowohl kognitive als auch motorische, personale und soziale Kompetenzen.

Im Bereich der Mediennutzung können nach der Intervention laut Erzieherinnen 66,7\% der Kinder den Computer selbstständiger nutzen, 55,2\% können selbstständiger mit der Digitalkamera umgehen und 34,8\% mit Büchern (vgl. Abb. 6). 


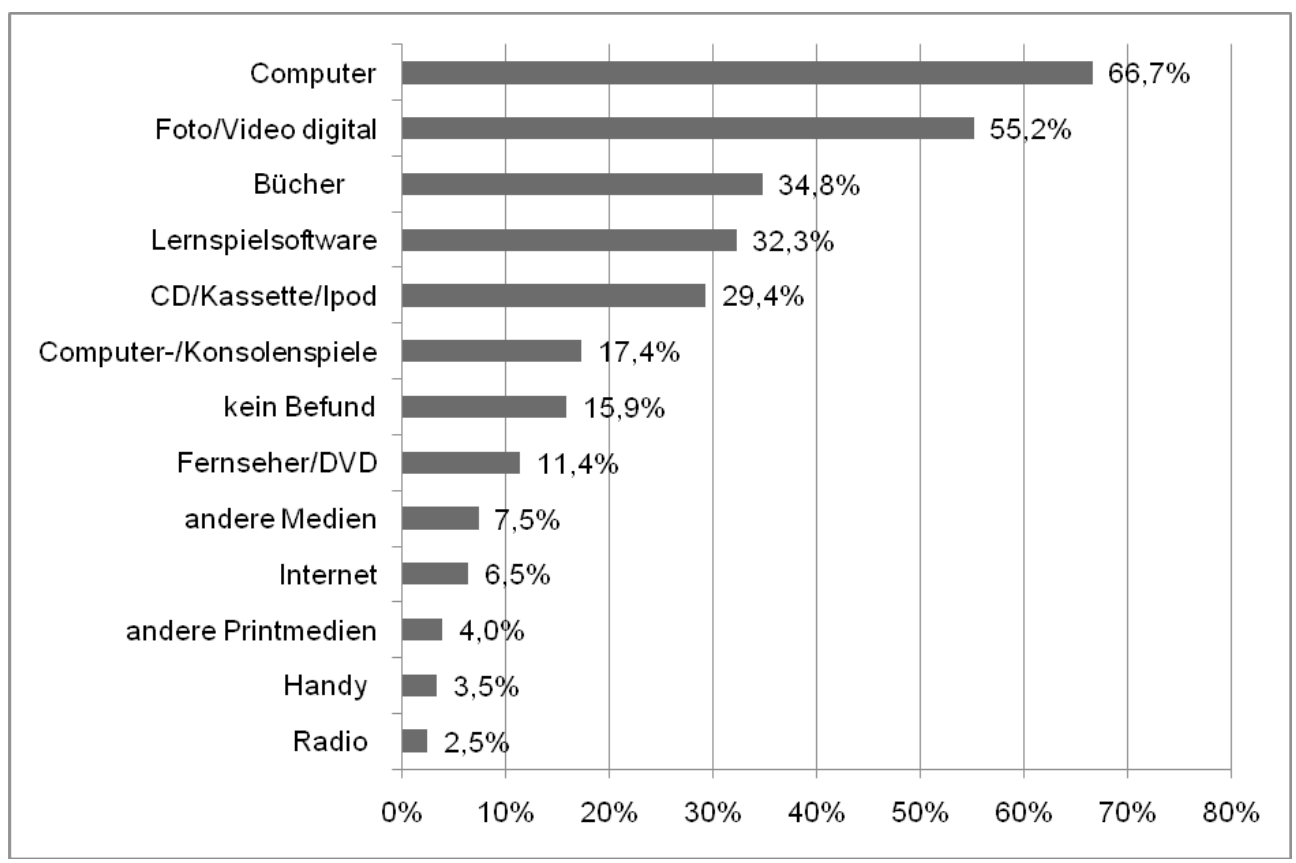

Abbildung 6: Selbstständigere Mediennutzung der Kinder nach Auskunft der Erzieherinnen $(\mathrm{N}=201)$

Generell haben 35\% der Kinder ihre technischen Fähigkeiten durch das Projekt erweitert und rund $24 \%$ der Erzieherinnen und Eltern gaben an, dass das Kind seit dem Projekt mit technischen Geräten sorgfältiger umgeht. Gleich viele Erzieherinnen und Eltern (20\%) stellen bei den Kindern eine Erweiterung des Verfügungswissens im technischen Bereich fest. Einem Lernspiel konzentrierter folgen können 22\% (Erzieherinnen) bzw. 21\% (Eltern) der Kinder, bei Bilderbüchern 14\%.

Bei 12\% (Eltern) bzw. 15\% (Erzieherinnen) der Kinder konnte eine Verbesserung des sprachlichen Ausdrucks festgestellt werden. Bei 14,2\% (Erzieherinnen) der Kinder wurde eine Verbesserung des Sozialverhaltens bemerkt. Sie zeigen sich offener und aufgeschlossener gegenüber Neuem und gehen im sozialen Miteinander rücksichtsvoller um. Die Erzieherinnen machten die Erfahrung, dass Kinder kontaktfreudiger wurden oder anderen Kindern halfen. Auch die personale Kompetenz einiger Kinder wurde durch wachsendes Selbstbewusstsein im Projekt sukzessive gestärkt. Allerdings ist auch hier bei einzelnen Werten Vorsicht geboten, da die normale Entwicklungsdynamik der untersuchten Altersgruppe nicht unterschätzt werden darf.

In Summe profitieren nach Angaben der Erzieherinnen 80\% der teilnehmenden Kinder vom Projekt. Für knapp die Hälfte davon hatte das Projekt eine besonders hohe pädagogische Bedeutung. Stärker profitiert haben nach Wahrnehmung der Erzieherinnen Jungen. Der geringste Kompetenzzuwachs wurde den Mädchen tür- 
kischer Herkunft attestiert. Bei 34\% dieser Gruppe sind den Erzieherinnen keine Kompetenzveränderungen aufgefallen. Für $27 \%$ der türkischen Mädchen wurde das Projekt von Erzieherinnen dementsprechend als mangelhaft bis ungenügend eingeschätzt. Eine mittlere Bedeutung hatte es für $45 \%$ dieser Gruppe und für $28 \%$ wurde es als sehr positiv bewertet. Im Vergleich hierzu schätzten die Erzieherinnen die Bedeutung des Projekts für $55 \%$ der Jungen mit türkischem Migrationshintergrund als sehr positiv ein. An dieser Stelle lassen die noch ausstehenden Ergebnisse der teilnehmenden Beobachtungen und die Auswertung der Puppet Interviews interessante Kontrollmöglichkeiten erwarten.

Dem entsprechen die Antworten der Eltern türkischer Herkunft, die bei $44 \%$ ihrer Töchter keiner Veränderung festgestellt haben wollen. Im Vergleich hierzu haben $76 \%$ der türkischen Eltern eine Veränderung im Kompetenzbereich ihrer Söhne festgestellt. Generell haben Eltern im Vergleich zu Erzieherinnen seltener eine Veränderung ihrer Kinder wahrgenommen.

Im Bereich der Medienkompetenz tendieren knapp 64\% der Eltern zu der Annahme, dass ihr Kind nach dem Projekt mehr über Medien weiss als zuvor. 14\% stellen diesbezüglich keine Veränderung fest. Vielfältigere Medien nutzen können 39,8\% der Kinder und mit Medien selbstständiger gestalten 53,2\%. Anschaffungswünsche bezüglich Medien haben 49,5\% der Eltern festgestellt. Einzelne Eltern konnten auch beobachten, dass ihr Kind bewusster und sensibler mit Medien umgeht, es wissbegieriger geworden ist sowie ein verstärktes Interesse am selbstständigen Umgang mit PC und anderen Medien entwickelt hat.

- Medienhandlungen in der Familie nach Intervention

Auf die Frage, ob sich etwas im gemeinsamen Medienverhalten in der Familie seit dem Projekt verändert hätte, gaben 38,1\% der Eltern ( $N=97)$ an, Medien häufiger gemeinsam mit dem Kind zu nutzen. 24,7\% der Kinder dürfen Medien seither selbstständiger nutzen und $11,3 \%$ der Kinder dürfen nun Medien nutzen, die sie davor nicht nutzen durften. 20,6\% der Eltern gaben an, seit dem Projekt stärker auf die Medieninhalte zu achten, die das Kind interessiert und nutzt. 9,3\% der Eltern sprechen mit ihren Kindern häufiger über Medien und 6,2\% informieren sich stärker über Medienerziehung. 7,2\% der Eltern haben inzwischen die Mediennutzung ihres Kindes eingeschränkt. 39,2\% der Eltern schliesslich haben keine Veränderung des familiären Medienverhaltens festgestellt.

\section{Schlussfolgerungen}

Die Ergebnisse der ersten Erhebungswelle bestätigen und verstärken die Befunde anderer Studien (Six u. a. 1998; Six u. Gimmler 2007; Marci-Boehncke u. Rath 2007b; Schneider u. a. 2010). Zum einen wird deutlich, dass Erzieherinnen oft ein unklares und inadäquates Verständnis von Medienerziehung und der Wirkungen 
des kindlichen Medienumgangs haben. Zum anderen wurde die Machbarkeit für Medienarbeit stark an den strukturellen Rahmenbedingungen der Einrichtungen bemessen. Diese subjektive Einschätzung geht a) auf einen einseitigen Medienbegriff zurück, der nur elektronische Medien als Medien wahrnimmt, und b) auf ein mangelndes Verständnis integrierter Medienarbeit, das die Medien als zusätzliches Thema und nicht als pädagogisch gestaltete Handlungsumgebung versteht. So sehen in der Eingangserhebung ein Drittel der Erzieherinnen für die Medienthematik in der Frühen Bildung keinen Problematisierungsbedarf und unterschätzen somit - da ist sich die Forschung einig - die Relevanz, v. a. auch von elektronischen Medien, in dieser Altersgruppe. Ebenso viele Erzieherinnen sprechen Medienkritik im Zusammenhang mit Medienerziehung keine bedeutende Rolle zu. Allerdings will fast die Hälfte ein Gefahrenpotenzial erkennen, das von Medien ausgehen kann. Die grössten Schwierigkeiten für die Medienarbeit sehen Erzieherinnen mitunter in der Betreuungsrelation von Erzieherinnen zu Kindern, den ökonomischen Ressourcen wie der Medienausstattung und dem Zeitfaktor. Die Ergebnisse zur Medienausstattung zeigen jedoch, dass die Kitas bereits vor Projektbeginn medial verhältnismässig gut ausgestattet waren, allerdings ein Teil der Erzieherinnen überhaupt nicht wusste, welche Medien in der eigenen Einrichtung zur Verfügung stehen, um mit den Kindern zu arbeiten. Darauf lässt zumindest die grosse Differenz der Angaben der befragten Erzieherinnen in den gleichen Einrichtungen zur Medienausstattung schliessen. Die Relevanz einer frühen Medienerziehung ist weder den Erzieherinnen noch Eltern wirklich bewusst.

Wie auch Ergebnisse aus der jährlich erscheinenden KIM-Studie (MPFS 2010) vermuten lassen und Daten aus weiteren Studien belegen (Feierabend u. Mohr 2004; Marci-Boehncke u. a. 2009; EOA 2011), nutzen bereits Kinder im Kita-Alter eine breite Medienpalette. Der PC und das Internet gehören längst nicht mehr zu den Randerscheinungen, sondern spielen bereits im Alltag von 4- und 5-Jährigen für etwa ein Drittel eine zentrale Rolle.

Dementsprechend kann die Kita als erste und früheste öffentliche Bildungsinstitution nicht mehr - wie noch in den 1990er Jahren (vgl. z. B. Six u. a. 1998, 118-134) - als «medienfreier Raum» definiert werden. Vielmehr muss, ausgehend von der medial verfassten Lebenswelt der Kinder, bereits in der Frühen Bildung eine Medienkompetenzvermittlung realisiert werden, die rezeptive Medienerfahrungen der Kinder auffängt und produktive Möglichkeiten verantwortungsbewusst erweitert. Gängige bewahrpädagogische Annahmen wie die These, Medien machten die Kinder aggressiv und ängstigen, kann nicht nur bereits nach der ersten Erhebungswelle durch Aussagen der Eltern entkräftet werden, sondern wird auch aus unserer bisherigen Forschung nicht bestätigt. Entgegen einer weiteren gängigen These, Kinder würden vor dem PC vereinsamen, zeigen die Ergebnisse der Abschlussbefragung der Erzieherinnen deutlich, dass Kinder - ähnlich wie Jugendliche auch 
(vgl. Barthelmes u. Sander 2001; Marci-Boehncke u. Rath 2007a) - den PC am liebsten mit Freunden und anderen Kindern im Spiel teilen und nur ein kleiner Teil der Kinder das Medium am liebsten alleine nutzt. Das Hybridmedium Computer hat neben Informations- und Gestaltungsfunktion auch eine Kommunikationsfunktion und ist selbst Ort sozialen Kontakts.

Deutlich wird, dass die bewahrpädagogischen Einstellungen der Erzieherinnen vor allem aus Mangel an Wissen über Medien und Mangel an eigenen Erfahrungen mit Medien resultieren. Allerdings zeigen die Ergebnisse der ersten Evaluation, dass eine Intervention, die die Erzieherinnen kontinuierlich begleitet, Abhilfe schafft. Wie bereits vermutet, fördert der Umgang mit einer breiten Medienpalette auch eine breite Palette an Kompetenzen. Die Arbeit mit Medien wirkt sich nicht nur gewinnbringend auf technische und kognitive Kompetenzen aus, sondern auch positiv auf das Sozialverhalten der Kinder. Medien bekommen Integrationsfunktion und auch die personale Kompetenz konnte in vielen Fällen gestärkt werden. Kinder, die bislang Schwierigkeiten hatten, sich in der Kita zu beweisen, gewinnen an Selbstbewusstsein und können hier «Experten» sein.

Zudem stieg nicht nur das Interesse an elektronischen Medien, sondern die Begeisterung an Printmedien und den zu ihrer Rezeption benötigten Kompetenzen nahm ebenfalls zu. Der Computer ist kein Konkurrenzmedium zum Buch, vielmehr lassen sich in einer integrativen Medienarbeit durch eine breite Medienpalette literarische und mediale Kompetenzen gemeinsam fördern. Übergreifende Medienarbeit fördert auch das Interesse an Büchern.

Eltern - das zeigen auch die Ergebnisse - haben ihre Verantwortung für die Mediensozialisation der Kinder häufig noch nicht ausreichend realisiert. Denn obgleich sich in $60 \%$ der Elternhäuser, nach Angaben der Eltern, das familiäre Medienverhalten verändert haben soll, nehmen die entscheidenden Aspekte der Medienerziehung auch nach der Intervention einen zu geringen Raum ein. So kommt die Anschlusskommunikation über Medien und Medieninhalte mit knapp $10 \%$ deutlich zu kurz und auch ein kritischer Medienumgang durch schärferen Blick auf die Medieninhalte und Medieninteresse der Kinder bleibt in vielen Haushalten bislang aus. Das Interesse an Medienerziehung konnte bei den Eltern nur geringfügig gesteigert werden - gerade einmal $6 \%$ informieren sich stärker über Medienerziehung. 


\section{Literatur}

Barthelmes, Jürgen und Ekkehard Sander. 2001. Erst die Freunde, dann die Medien. Medien als Begleiter in Pubertät und Adoleszenz: Medienerfahrungen von Jugendlichen. Bd. 2. München, Opladen: DJI, Leske + Budrich.

Baumert, Jürgen, Kai Maaz, Cornelia Gresch, Nele McElvany, Yvonne Anders, Kathrin Jonkmann, Marko Neumann und Rainer Watermann. 2010. «Der Übergang von der Grundschule in die weiterführende Schule - Leistungsgerechtigkeit und regionale, soziale und ethnisch-kulturelle Disparitäten: Zusammenfassung der zentralen Befunde.» In Der Übergang von der Grundschule in die weiterführende Schule. Leistungsgerechtigkeit und regionale, soziale und ethnischkulturelle Disparitäten. Bildungsforschung Bd. 34, hrsg. v. Jürgen Baumert, Cornelia Gresch, Kai Maaz und Nele McElvany, 5-21. Bonn, Berlin: Bundesministerium für Bildung und Forschung.

Becker, Birgit und Nicole Biedinger. 2006. «Ethnische Bildungsungleichheit zu Schulbeginn.» Kölner Zeitschrift für Soziologie und Sozialpsychologie 58 (4): 660-684.

Becker, Rolf und Wolfgang Lauterbach. 2004. «Vom Nutzen vorschulischer Kinderbetreuung für Bildungschancen.» In Bildung als Privileg? Erklärungen und Befunde zu den Ursachen der Bildungsungleichheit, hrsg. v. Rolf Becker und Wolfgang Lauterbach, 127-159. Wiesbaden: VS Verlag.

Bourdieu, Pierre. 1983. «Ökonomisches Kapital, kulturelles Kapital, soziales Kapital.» In Soziale Ungleichheiten (Soziale Welt, Sonderband 2), hrsg. v. Reinhard Kreckel, 183-198. Göttingen: Schwartz.

Bourdieu, Pierre. 1987. Sozialer Sinn. Kritik der theoretischen Vernunft. Frankfurt am Main: Suhrkamp.

Bourdieu, Pierre. 1992. Rede und Antwort. Frankfurt am Main: Suhrkamp.

Bourdieu, Pierre. 1993. Soziologische Fragen. Frankfurt am Main: Suhrkamp.

Bourdieu, Pierre. 1982. Die feinen Unterschiede: Kritik der gesellschaftlichen Urteilskraft. Frankfurt am Main: Suhrkamp.

Bourdieu, Pierre und Loïc J. D. Wacquant. 1996. Reflexive Anthropologie. Frankfurt am Main: Suhrkamp.

Diefenbach, Heike. 2007. Kinder und Jugendliche aus Migrantenfamilien im deutschen Bildungssystem. Erklärungen und Befunde. Wiesbaden: VS Verlag.

Dortmunder Statistik. 2011. Jahresbericht, Dortmunder Statistik 2011, Bevölkerung. Nachdruck, hrsg. v. Stadt Dortmund: http://www.dortmund.de/media/ downloads/pdf/statistik/veroeffentlichungen/jahresberichte/bevoelkerung_1/ Nummer_193_-_Bevoelkerung_2011.pdf.

Ehmke, Timo und Nina Jude. 2010. "Soziale Herkunft und Kompetenzerwerb.» PISA 2009. Bilanz nach einem Jahrzehnt, hrsg. v. Eckhard Klieme, Cordula 
Artelt, Johannes Hartig, Nina Jude, Olaf Köller, Manfred Prenzel, Wolfgang Schneider und Petra Stanat, 231-254. Münster: Waxmann.

EOA. 2011. Klick mich! Wie man die Herzen der jungen User erobert! Erfolgreiche Emotionalisierung im Online-Marketing für Kids und Teens. Mönchengladbach: Elements of Art.

Feierabend, Sabine und Inge Mohr. 2004. «Mediennutzung von Klein- und Vorschulkindern. Ergebnisse der ARD/ZDF-Studie «Kinder und Medien 2003〉.» Media Perspektiven (9): 453-461.

Flick, Uwe. 2004. Triangulation: Eine Einführung. Wiesbaden: VS Verlag.

Flick Uwe. 2005. «Triangulation in der qualitativen Forschung.»In Qualitative Forschung. Ein Handbuch. 4. Auflage, hrsg. v. Uwe Flick, Ernst von Kardorff und Ines Steinke, 309-318. Reinbek bei Hamburg: Rowohlt Taschenbuch Verlag.

Glaser, Barney G. und Anselm Strauss. 1998. Grounded Theory: Strategien qualitativer Forschung. Göttingen: H. Huber.

Katz, Lilian G. und Sylvia C. Chard. 2000. Engaging children's minds: The project approach. 2nd edition. Stamford, CT: Ablex.

Kleining, Gerhard. 1994. Qualitativ-heuristische Sozialforschung. Schriften und Theorie und Praxis. Hamburg-Harvestehude: Fechner: http://www.ssoar.info/ ssoar/files/2010/1160/qualitativ_heur.pdf.

Kreyenfeld, Michaele. 2004. "Soziale Ungleichheit und Kinderbetreuung. Eine Analyse der sozialen und ökonomischen Determinanten der Nutzung von Kitas.» In Bildung als Privileg? Erklärungen und Befunde zu den Ursachen der Bildungsungleichheit, hrsg. v. Rolf Becker und Wolfgang Lauterbach, 99-125. Wiesbaden: VS Verlag.

Krotz, Friedrich. 2005. Neue Theorien entwickeln: Eine Einführung in die Grounded Theory, die Heuristische Sozialforschung und die Ethnographie anhand von Beispielen aus der Kommunikationsforschung. Köln: Halem Verlag.

Marci-Boehncke, Gudrun und Matthias Rath. 2007a. Jugend - Werte - Medien: Die Studie. Weinheim: Beltz.

Marci-Boehncke, Gudrun und Matthias Rath. 2007b. Medienkompetenz für Erzieherlnnen: Ein Handbuch für die moderne Medienpraxis in der Frühen Bildung. München: kopaed.

Marci-Boehncke, Gudrun, Marion Weise und Matthias Rath. 2009. «Kinder auf die Spuren bringen > Medien- und Lesekompetenz in der Elementarbildung.» In Leseförderung im Kindergarten- und Grundschulalter. Wissenschaftlicher Diskurs und praktische Initiativen, hrsg. v. Stefan Salamonsberger, Normann Stricker und Volker Titel, 151-166. Erlangen: Friedrich-Alexander-Universität/ Buchwissenschaft.

Marci-Boehncke, Gudrun. 2009. «Hallo, SpongeBob!) oder: Kindliche Mediennutzung im Medienverbund.» In Kinder im Blick: Medienkompetenz statt Me- 
dienabstinenz. Dieter Baacke Preis - Handbuch 4, hrsg. v. Jürgen Lauffer und Renate Röllecke, 37-49. Bielefeld: GMK Gesellschaft für Medienpädagogik und Kommunikationskultur: http://www.dieterbaackepreis.de/fileadmin/pdf/ dieter_baacke_b\%C3\%BCcher/db4_marci-boehncke2009.pdf.

Marci-Boehncke, Gudrun. 2011. "Verantwortungskooperationen zur Medienbildung: Zwischen Selbstsozialisation und Erziehungsverantwortung - Ein Blick auf die Frühe Bildung.»In Kooperative Bildungsverantwortung. Sozialethische und pädagogische Perspektiven auf «Educational Governance», hrsg. v. Marianne Heimbach-Steins und Gerhard Kruip, 143-160. Bielefeld: W. Bertelsmann Verlag.

MPFS. 2010. KIM-Studie 2010. Kinder und Medien. Computer und Internet: Basisuntersuchung zum Medienumgang 6- bis 13-Jähriger in Deutschland, hrsg. v. Medienpädagogischen Forschungsverbund Südwest. Stuttgart: Medienpädagogischer Forschungsverbund Südwest.

Mikos, Lothar. 2005. "Teilnehmende Beobachtung.» In Qualitative Medienforschung: Ein Handbuch, hrsg. v. Lothar Mikos und Claudia Wegener, 315-323. Konstanz: UVK.

MSJK NRW. 2003. Bildungsvereinbarung NRW - Fundament stärken und erfolgreich starten. Hrsg. v. Ministerium für Schule, Jugend und Kinder des Landes Nordrhein-Westfalen, Düsseldorf: https://services.nordrheinwestfalendirekt. de/broschuerenservice/download/1343/bildungsvereinbarung.pdf.

MSW NRW/MFKJKS NRW 2011. Kinder. Mehr Chancen durch Bildung von Anfang an - Entwurf - Grundsätze zur Bildungsförderung für Kinder von 0 bis 10 Jahren in Kindetageseinrichtungen und Schulen im Primarbereich in NordrheinWestfalen. Hrsg. v. Ministerium für Schule und Weiterbildung und Ministerium für Familie, Kinder, Jugend, Kultur und Sport des Landes Nordrhein-Westfalen. Düsseldorf: http://www.mfkjks.nrw.de/web/media_get.php?mediaid=17282\&fi leid $=51011 \&$ sprachid $=1$.

Rabe-Kleberg, Ursula. 2010. «Bildungsarmut von Anfang an? Über den Beitrag des Kindergartens im Prozess der Reproduktion sozialer Ungleichheit.» Bildungsungleichheit revisited. Bildung und soziale Ungleichheit vom Kindergarten bis zur Hochschule, hrsg. v. Heinz-Hermann Krüger, Ursula Rabe-Kleberg, RolfTorsten Kramer uns Jürgen Budde, 45-54. Wiesbaden: VS Verlag.

Rath, Matthias. 2011. "Übergänge sind immer. Anthropologische Überlegungen zu einem pädagogischen Thema. » Friedrich Jahresheft (39), Themenheft «Übergänge», 10-13.

Schneider, Beate, Helmut Scherer, Nicole Gonser und Annekaryn Tiele. 2010. Medienpädagogische Kompetenz in Kinderschuhen. Eine empirische Studie zur Medienkompetenz von Erzieherinnen und Erziehern in Kindergärten. Berlin: Vistas. 
Six, Ulrike, Christoph Frey und Roland Gimmler. 1998. Medienerziehung im Kindergarten. Theoretische Grundlagen und empirische Befunde. Schriftenreihe Medienforschung der Landesanstalt für Rundfunk Nordrhein-Westfalen Bd. 28. Opladen: Leske + Budrich.

Six, Ulrike und Roland Gimmler. 2007. Die Förderung von Medienkompetenz im Kindergarten: Eine empirische Studie zu Bedingungen und Handlungen der Medienerziehung. Schriftenreihe Medienforschung der Landesanstalt für Medien Nordrhein-Westfalen Bd. 57. Berlin: Vistas.

Six, Ulrike. 2010. «Mediensozialisation und Medienbildung im Kindergarten.»In Handbuch Mediensozialisation. Hrsg. v. Ralf Vollbrecht und Claudia Wegener, 201-207. Wiesbaden: VS Verlag.

Weise, Marion. 2008. «Der Kindergarten wird zum 〈Forschungsort〉- Das Puppet Interview als Forschungsmethode für die Frühe Bildung.» Ludwigsburger Beiträge zur Medienpädagogik (11): http://www.ph-ludwigsburg.de/fileadmin/ subsites/1b-mpxx-t-01/user_files/Online-Magazin/Ausgabe11/Weise11.pdf.

Weise, Marion. 2011. «Kids konvergent - Wie Vorschulkinder konvergierende Medien (für sich) nutzen.» Medienkonvergenz im Deutschunterricht. Schriftenreihe Jahrbuch Medien im Deutschunterricht 2010, hrsg. v. Gudrun Marci-Boehncke und Matthias Rath, 50-69. München: kopaed. 